\title{
Characteristics of Highly Birefringent Photonic Crystal Fiber with Defected Core and Equilateral Pentagon Architecture
}

\author{
Fei Yu, Zhenpeng Wang, Wenhao Yang, and Chongyang Lv \\ College of Automation, Harbin Engineering University, No. 145 Nantong Street, Harbin 150001, China \\ Correspondence should be addressed to Zhenpeng Wang; wzpstudy@126.com
}

Received 10 May 2016; Revised 29 July 2016; Accepted 7 August 2016

Academic Editor: Vasily Spirin

Copyright ( 2016 Fei Yu et al. This is an open access article distributed under the Creative Commons Attribution License, which permits unrestricted use, distribution, and reproduction in any medium, provided the original work is properly cited.

\begin{abstract}
A novel high birefringence and nearly zero dispersion-flattened photonic crystal fiber (PCF) with elliptical defected core (E-DC) and equilateral pentagonal architecture is designed. By applying the full-vector finite element method (FEM), the characteristics of electric field distribution, birefringence, and chromatic dispersion of the proposed E-DC PCF are numerically investigated in detail. The simulation results reveal that the proposed PCF can realize high birefringence, ranging from $10^{-3}$ to $10^{-2}$ orders of magnitude, owing to the embedded elliptical air hole in the core center. However, the existence of the elliptical air hole gives rise to an extraordinary electric field distribution, where a V-shaped notch appears and the size of the V-shaped notch varies at different operating wavelengths. Also, the mode field diameter is estimated to be about $2 \mu \mathrm{m}$, which implies the small effective mode area and highly nonlinear coefficient. Furthermore, the investigation of the chromatic dispersion characteristic shows that the introduction of the elliptical air hole is helpful to control the chromatic dispersion to be negative or nearly zero flattened over a wide wavelength bandwidth.
\end{abstract}

\section{Introduction}

PCFs, also called holey fibers or microstructured optical fibers, have received extensive attention since they were firstly fabricated and reported by Knight et al. [1]. One of the most appealing features of PCFs is their high flexibility based on the particular geometry of their refractive index distribution. In order to meet requirements for different applications, researchers have designed kinds of PCFs such as high birefringence PCF [2-6], ultraflattened chromatic dispersion PCF $[7,8]$, and high nonlinear coefficient PCF $[9,10]$. The modal birefringence of polarization maintaining PCFs is reported to be $10^{-3}$ orders of magnitude which is one order higher than that of conventional PMFs $[3,11]$. Therefore, high birefringence PCFs are considered to be good candidates for sensing applications, where light is required to maintain a linear polarization state. Meanwhile, the task of controlling the chromatic dispersion is a very important problem in designing practical optical communication systems, dispersion controllers, or nonlinear systems [12].

In conventional polarization maintaining index-guiding PCFs, an absolute solid core (SC) with greater refractive index is required to confine the propagating light, and high birefringence is realized by having different air hole diameters in the cladding along the two orthogonal axes or by asymmetric core design $[2-6,11,13]$. Recently, it is interesting to find that a type of index-guiding PCF characterized with a circular air hole defected core (C-DC) is reported [14-16], which is different from the absolute SC in the conventional indexguiding PCFs. Saitoh et al. [14] firstly put forward the concept of C-DC PCF, in which a circular air hole was embedded in the core region so as to freely control the chromatic dispersion. Thereafter, on the basis of this interesting idea, a few C-DC PCF microstructures were proposed and investigated. Yin and Xiong [15] designed a C-DC PCF with the hybrid cladding of square and circular air holes, and numerical analysis showed that the low confinement loss and nearly zero ultraflattened chromatic dispersion could be realized. Then, Zhang et al. [16] proposed a square-lattice C-DC PCF, where the embedded circular air hole in the center was used to adjust the waveguide dispersion to offset the material dispersion for obtaining nearly zero ultraflattened chromatic dispersion. However, in these above-mentioned DC PCFs, it is circular air holes that are introduced into the core region only for the 


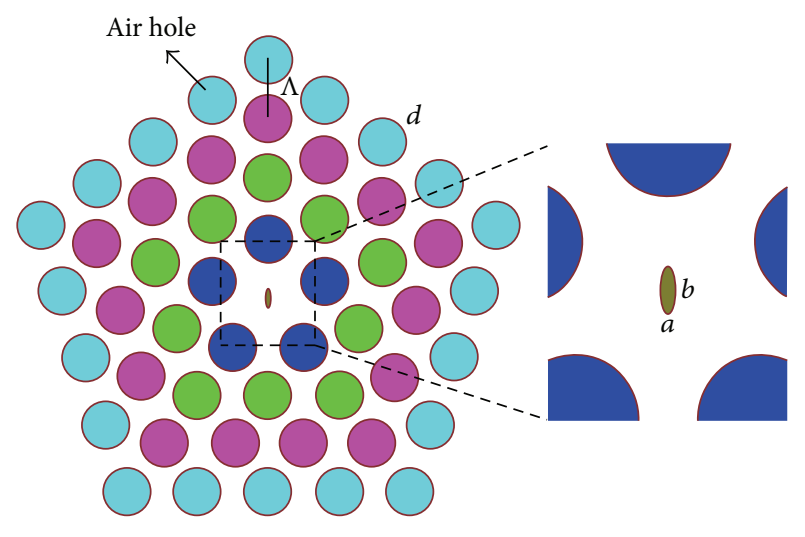

FIgURE 1: The cross section of the proposed PCF.

purpose of controlling the chromatic dispersion. Besides, the existence of DC will have a certain influence on the mode field distribution, which, to our knowledge, has not been investigated in detail.

In this paper, from the perspective of improving modal birefringence and simultaneously controlling the chromatic dispersion, based on the C-DC idea, we consider introducing an elliptical air hole defected core (E-DC) in the core center to enhance the structural asymmetry. Also, it is confirmed in [17-19] that higher birefringence and less power loss could be obtained in the equilateral pentagonal PCF than in the hexagonal PCF. Therefore, a highly birefringent PCF with equilateral pentagon cladding and $\mathrm{E}-\mathrm{DC}$ is designed, and the accompanying influences of E-DC on electric field distribution, birefringence, and chromatic dispersion are numerically investigated by full-vector FEM. Simulation results reveal that the electric fields of $x$ and $y$ polarization modes in the proposed E-DC PCF possess V-shaped notches, not like the Gaussian distribution in the absolute SC PCFs, in the core region due to the embedded elliptical air hole; meanwhile the mode field diameter is estimated to be about $2 \mu \mathrm{m}$, which implies highly nonlinear coefficient. Furthermore, the modal birefringence of the E-DC PCF can be up to $8 \times$ $10^{-3}$ at the operating wavelength of $1.55 \mu \mathrm{m}$, or even higher. In addition, the introduction of the E-DC is helpful to control the chromatic dispersion to be negative or nearly zero flattened over a wide wavelength range. The proposed high birefringence E-DC PCF may find important applications in the field of optical fiber communications, fiber lasers, and fiber sensors, and the added negative or flattened dispersion feature makes it suitable as a chromatic dispersion controller, a dispersion compensator, or a candidate for nonlinear optical applications.

\section{Theoretical Basis}

The transverse section of the proposed PCF is shown in Figure 1, where symbols $d$ and $\Lambda$ represent the diameter of air holes and pitch constant (hole to hole space) in the cladding and $a$ and $b$ are minor axis and major axis of the central elliptical air hole, respectively. The background material is assumed to be silica, and the refractive index as a function of wavelength can be obtained by using Sellmeier equation:

$$
n^{2}(\lambda)=1+\sum_{i=1}^{3} \frac{A_{i} \lambda^{2}}{\lambda^{2}-\lambda_{i}^{2}},
$$

where $\lambda$ is the incident wavelength and coefficients $A_{i}$ and $\lambda_{i}$ can be taken from [20].

To accurately model the proposed PCF, we adopt fullvector FEM with hybrid edge/node elements that can avoid spurious solutions for modeling curved boundaries [21, 22]. The full-vector FEM is capable of flexibly and accurately dealing with the complicated-structure PCF with arbitraryshape holes [23]. In the process of calculation, the effective refractive indexes at incremental operating wavelengths are recorded. Then, the data postprocessing operation is implemented by using Matlab software.

The fiber birefringence reflects the polarization maintaining ability, and high birefringence can improve the system's accuracy and stability. The birefringence can be obtained by [2-6]

$$
B=n_{\mathrm{eff}}^{x}-n_{\mathrm{eff}}^{y}
$$

where $n_{\mathrm{eff}}^{x}$ and $n_{\mathrm{eff}}^{y}$ are effective refractive indexes of the two orthogonal polarization modes ( $x$-polarization and $y$ polarization modes) of the fundamental mode, respectively. The total chromatic dispersion can be obtained by the following equation $[12,15]$ :

$$
D(\lambda)=-\frac{\lambda}{c} \frac{d^{2} n_{\mathrm{eff}}(\lambda)}{d \lambda^{2}},
$$

where $c$ is the velocity of the propagation light in vacuum. It should be noted that the material dispersion has been considered during the calculation of the effective refractive index $n_{\text {eff }}$ by using (1). After the discrete values of $n_{\text {eff }}(\lambda)$ at different wavelengths are worked out, there may be two methods to calculate the second derivative for (3): one is that by the polynomial fitting we can get the functional relation between $n_{\text {eff }}(\lambda)$ and wavelength $\lambda$, and then the second derivative can be easily obtained; the other method adopted in this paper is directly via the differential form [16]:

$$
\begin{aligned}
D\left(\lambda_{i}\right)=-\frac{\lambda_{i}}{c} & \\
. & \frac{\Delta n_{\mathrm{eff}}\left(\lambda_{i+1}, \lambda_{i}\right) / \Delta \lambda(i+1, i)-\Delta n_{\mathrm{eff}}\left(\lambda_{i}, \lambda_{i-1}\right) / \Delta \lambda(i, i-1)}{0.5 \Delta \lambda(i+1, i-1)},
\end{aligned}
$$

where $\Delta n_{\text {eff }}\left(\lambda_{m}, \lambda_{n}\right)=n_{\text {eff }}\left(\lambda_{m}\right)-n_{\text {eff }}\left(\lambda_{n}\right), \Delta \lambda(m, n)=\lambda_{m}-$ $\lambda_{n}$, and $n_{\text {eff }}\left(\lambda_{m}\right)$ is the effective refractive index of the fundamental mode at the wavelength of $\lambda_{m}$.

\section{Simulation and Characteristics}

3.1. Electric Field Distribution Characteristics. It is well known that the modal field profile depends on the refractive index distribution across the optical waveguide cross section. For the conventional absolute SC PCF, the modal field profile matches the Gauss distribution, namely, that the most intense 


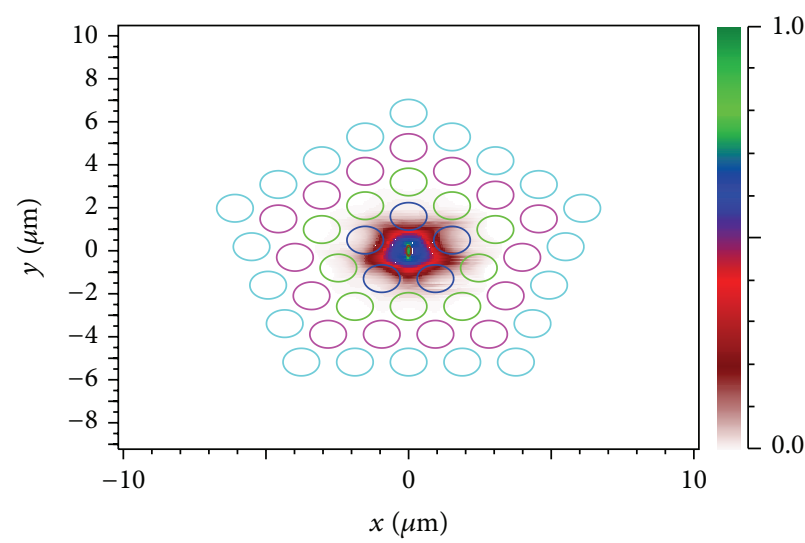

(a) $x$-polarized mode

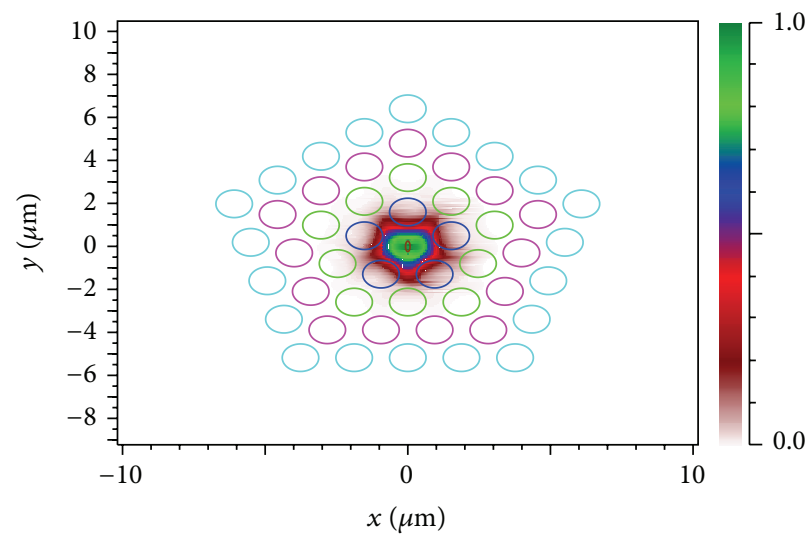

(b) $y$-polarized mode

FIGURE 2: The normalized electric field power at the operating wavelength of $1.55 \mu \mathrm{m}$ for (a) $x$-polarized mode and (b) $y$-polarized mode. The structural parameters are set as $\Lambda=1.6 \mu \mathrm{m}, d / \Lambda=0.8, a / \Lambda=0.1$, and $\eta=3$.

light appears at the core center. However, for the proposed PCF, the introduction of E-DC reduces the refractive index in the core center, which alters the characteristic of the modal field distributions. Figures 2(a) and 2(b) show the transverse electric field distributions of $x$-polarization and $y$ polarization modes at the operating wavelength of $1.55 \mu \mathrm{m}$, where the structural parameters are set as $\Lambda=1.6 \mu \mathrm{m}, d / \Lambda$ $=0.8, a / \Lambda=0.1$, and $\eta=3$. It can be obviously seen that the electric fields of $x$-polarization and $y$-polarization modes are well confined in the core region although little field power penetrates into the lattice near the core of the PCF. However, we can also observe that the modal power distributions between $x$-polarization and $y$-polarization modes are quite different in the core region. In order to further clearly demonstrate the difference, the curves of electric field amplitudes of $x$-polarization and $y$-polarization modes as a function of the horizontal distance along the minor axis direction are plotted in Figure 3. It can be found that both curves possess a Vshaped notch in the core region instead of the approximate Gaussian distribution like in SC PCFs. The power of $x$ polarization mode around the elliptical air hole increases sharply, and the maximum field intensity is concentrated in the center. However, for $y$-polarization mode, it does not exhibit such a behavior. $y$-polarized curve looks smoother in the core region, and its maximum locates between the central defect and the first air hole layer in the cladding.

The electric field distributions at different wavelengths for $x$-polarization and $y$-polarization modes are also investigated, as shown in Figure 4. We can see that the intensity in the center increases with the increase of wavelength, which means that the power gradually transfers from the surrounding silica into the central elliptical hole. For $x$ polarization mode, when the operating wavelength exceeds a certain length, the intensity in the elliptical air hole then becomes the maximum, while for $y$-polarization mode the maximum value always locates between the central defect and the first air hole layer in the cladding no matter how long the wavelength is. In addition, we can find that the diameter of the mode field is less than $2 \mu \mathrm{m}$, which implies that the proposed

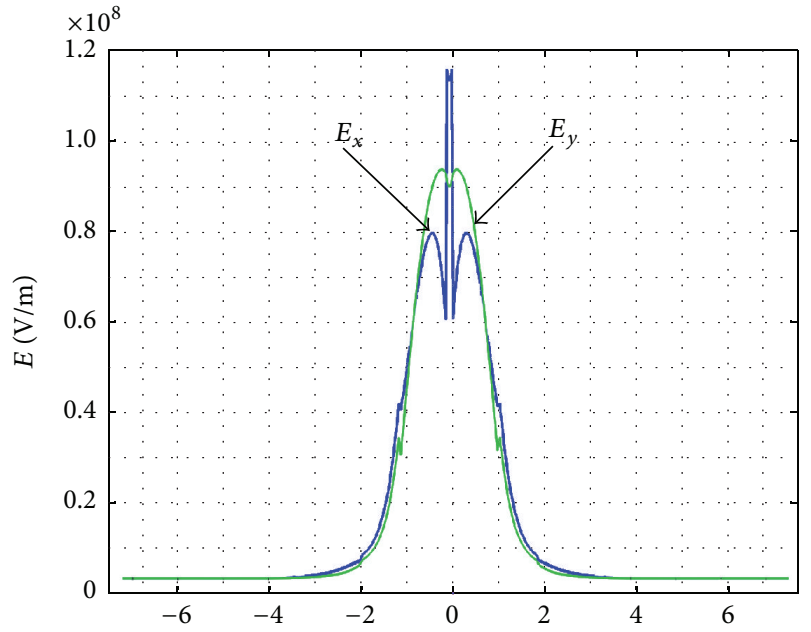

FIgURE 3: The electric field amplitundes for the two polarization modes at the wavelength of $1.55 \mu \mathrm{m}$ along the minor axis direction. The structural parameters are set as $\Lambda=1.6 \mu \mathrm{m}, d / \Lambda=0.8, a / \Lambda=0.1$, and $\eta=3$.

PCF has the small effective mode area and highly nonlinear coefficient.

3.2. Modal Birefringence Characteristics. One of the interesting characteristics of the proposed E-DC PCF is its strong birefringence. The formation of the required asymmetric microstructure comes from the insertion of the elliptical air hole in the core center. The modal birefringence can be obtained from (2), where the effective refractive indexes are computed by applying FEM. Before investigating the proposed E-DC PCF, we firstly evaluate the modal birefringence of the absolute SC PCF, namely, without the elliptical air hole in the core center. It can be found that the modal birefringence is as small as $10^{-6}$ orders of magnitude, owing to the weak asymmetry configuration.

Thereafter, the birefringence characteristics of the proposed E-DC PCF and the impacts of structural parameters 


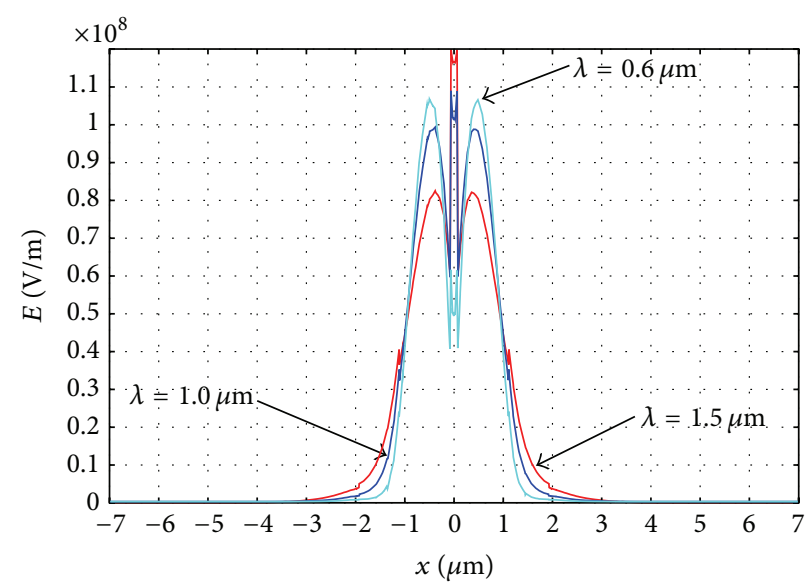

(a) $x$-polarized mode

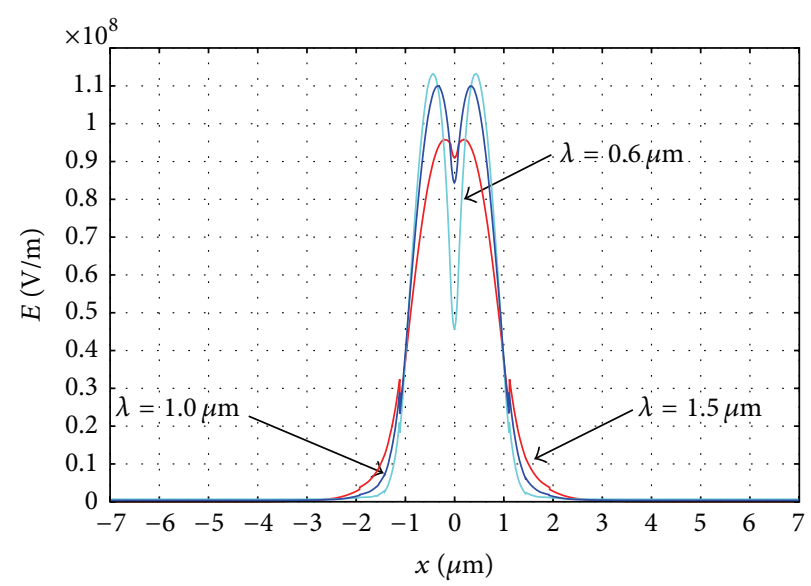

(b) $y$-polarized mode

FIGURE 4: The impact of the variation of wavelengths on the electric field amplitude for (a) $x$-polarized mode and (b) $y$-polarized mode.

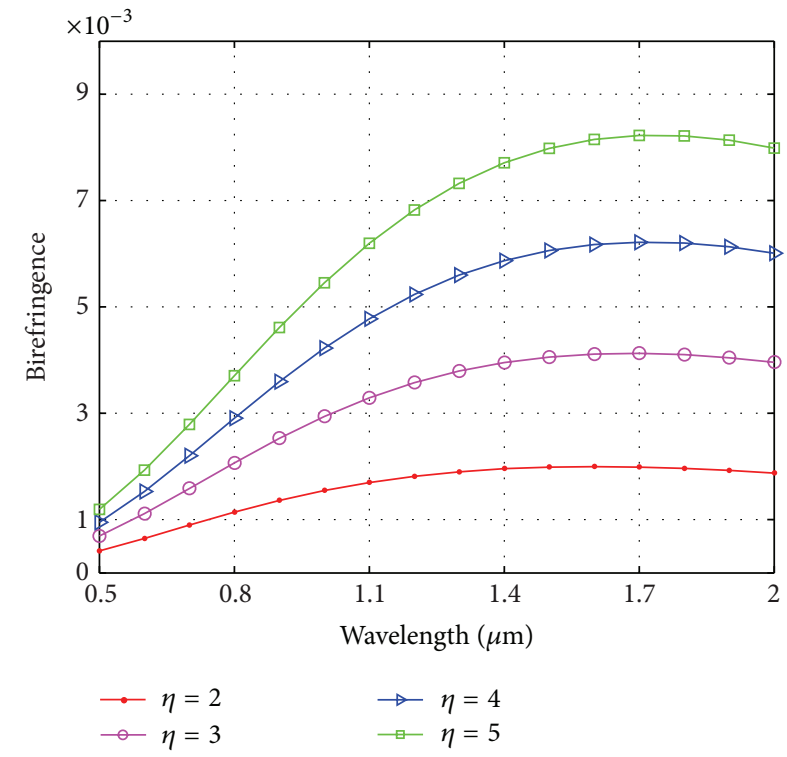

(a) Different values of $\eta$

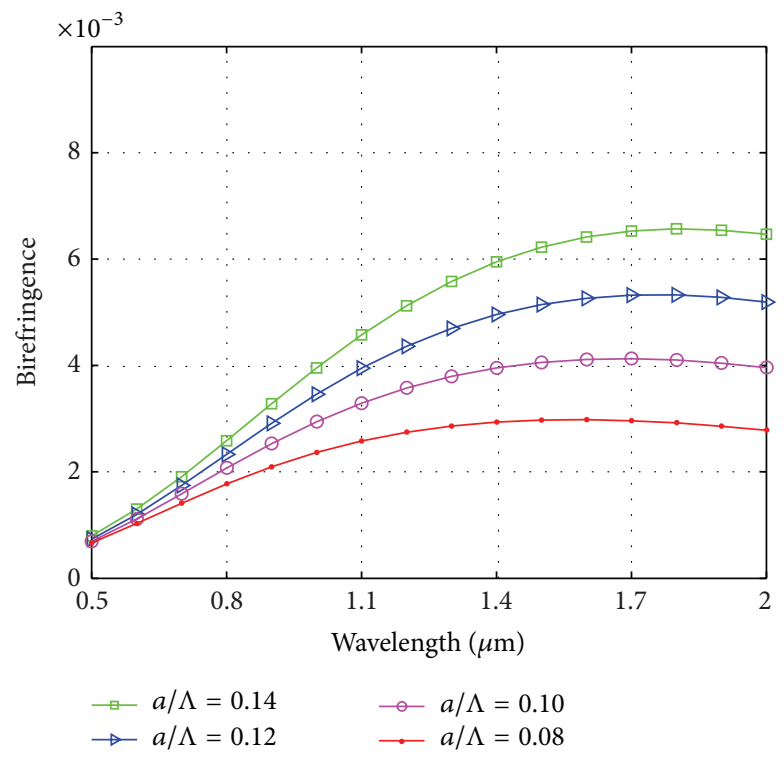

(b) Different values of $a / \Lambda$

Figure 5: Birefringence curves as a function of wavelength for the defected core PCF with different values of (a) $\eta$ at $a / \Lambda=0.1$ and (b) $a / \Lambda$ at $\eta=3$. The other design parameters are set as $\Lambda=1.6 \mu \mathrm{m}$ and $d / \Lambda=0.8$.

are investigated in detail. The modal birefringence in the E-DC PCF mainly results from the asymmetry in $x$ and $y$ directions due to the introduction of the elliptical air hole in the core center, and thus it is feasible to alter the size of the elliptical hole to strengthen or weaken the modal birefringence. Figures 5(a) and 5(b) show the modal birefringence as a function of wavelength with different designed parameter $\eta$ and normalized minor axis $a / \Lambda$, where fixed parameters $\Lambda$ and $d / \Lambda$ are set to be $1.6 \mu \mathrm{m}$ and 0.8 , respectively. It can be observed that the modal birefringence becomes larger with the increase of the value of $\eta$, and the birefringence value can be up to $8 \times 10^{-3}$ at the wavelength of $1.55 \mu \mathrm{m}$. Furthermore, Figure 5(b) indicates that enlarging the size of the minor axis $a$ can also strengthen the polarization maintaining ability.
Therefore, the proposed PCF has the ability to tune the birefringence up to $10^{-2}$ orders of magnitude with further optimizing parameters $\eta$ and $a$.

In addition, impacts of microadjustment of designed parameters lattice constant $\Lambda$ and normalized diameter $d / \Lambda$ on birefringence are also analyzed, as shown in Figure 6. It can be seen that each curve has a maximum point within wavelengths ranging from 0.6 to $2 \mu \mathrm{m}$. This is different from that of other PCFs in $[3-6,17-19]$, where the birefringence value increases monotonically with the increase of the wavelength. It can be also noticed that when the lattice constant $\Lambda$ decreases, the location of the maximum point shifts towards the short wavelengths but its value changes little. Therefore, we can adjust the lattice constant $\Lambda$ to get the 


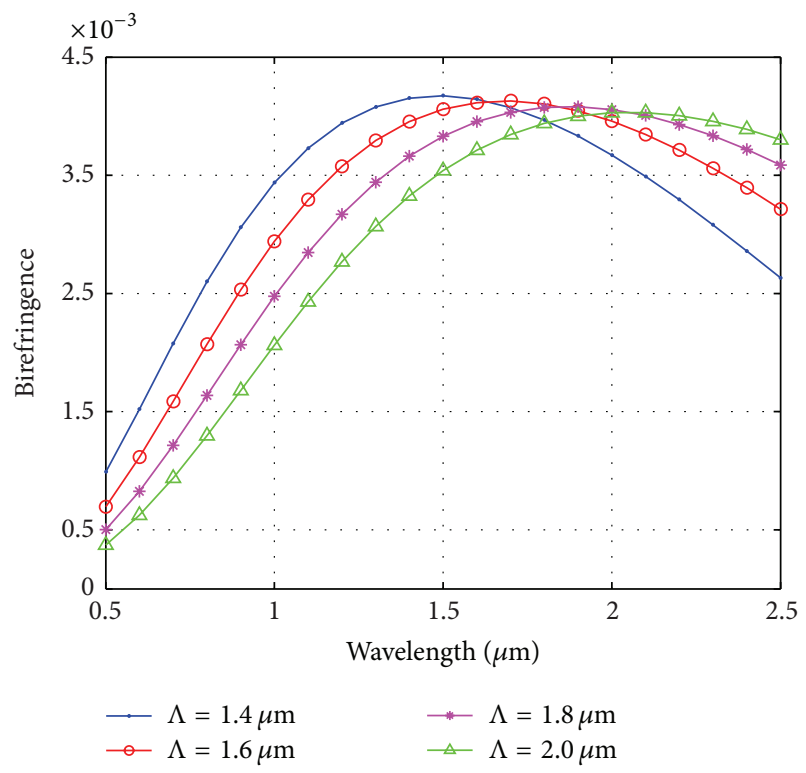

(a) Different values of $\Lambda$

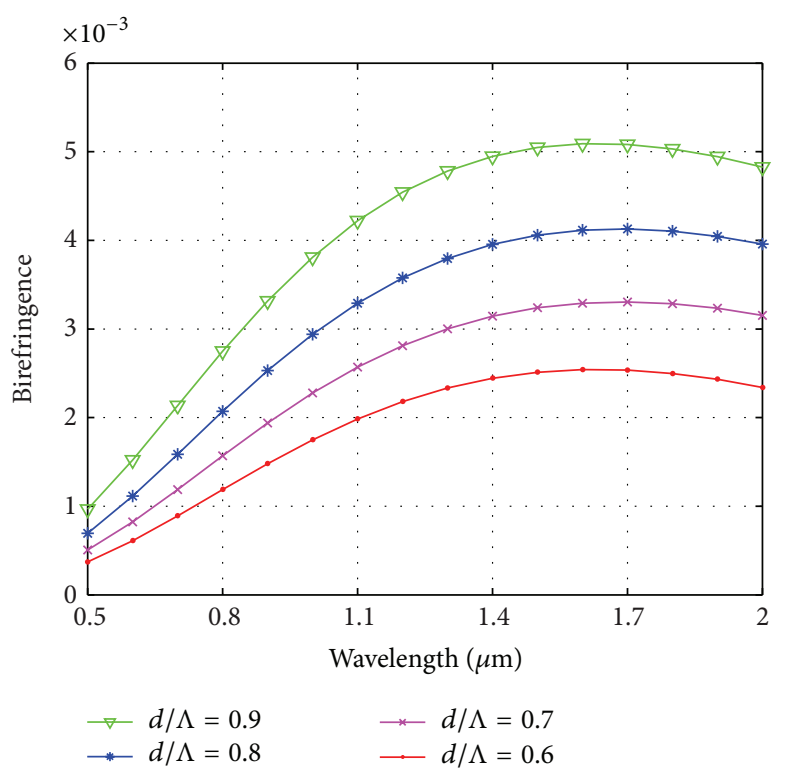

(b) Different values of $d / \Lambda$

FIGURE 6: The dependent relationship between birefringence and wavelength for the defected core PCF with different parameters: (a) lattice constant $\Lambda$ at $d / \Lambda=0.8$ and (b) normalized $d / \Lambda$ at $\Lambda=1.6 \mu \mathrm{m}$. The remaining parameters are set as $a / \Lambda=0.1$ and $\eta=3$.

maximum point to operate at a given wavelength. Figure 6(b) shows that increasing the diameter $d$ can also enlarge the modal birefringence in the whole wavelength range. The analysis about the influence of structural parameters on the birefringence for the E-DC PCF is useful for optimizing parameters for obtaining higher birefringence.

According to Figure 5(a), when the geometric parameters are set as $\Lambda=1.6 \mu \mathrm{m}, d / \Lambda=0.8, a / \Lambda=0.1$, and $\eta=5$, the modal birefringence is evaluated to be about $8 \times 10^{-3}$ at the operating wavelength of $1.55 \mu \mathrm{m}$, which strongly proves that the proposed structure is highly polarized. Compared with the equilateral pentagonal polarization maintaining PCF in [17-19], the proposed E-DC PCF exhibits higher birefringence behavior and the simple structure feature. Note that the values of parameters $d / \Lambda$ and $a / \Lambda$ cannot be too large, although increasing them can help increase the modal birefringence. This is because the large diameter $d$ can lead to lower effective refractive index of the cladding to induce the second-order mode in the PCF, while too large value of $a$ can cause the refractive index of the fundamental mode to be lower than the cladding index, which means that the fundamental mode will completely leak into the cladding.

3.3. Chromatic Dispersion Characteristics. PCFs possess the attractive property of great controllability in chromatic dispersion. The controllability of chromatic dispersion is of great importance for practical applications to dispersion compensation, optical communication systems, and nonlinear optics [12]. The chromatic dispersion profile can be easily controlled by varying the structural parameters. In order to explore the influence of the E-DC on the chromatic dispersion, it is required to make a comparison with the equilateral pentagonal SC PCF. Figure 7 shows the chromatic dispersion property of the SC PCF as a function of wavelength for different normalized diameter $d / \Lambda$ ranging from 0.6 to 0.9 in steps of 0.1. When the lattice constant $\Lambda$ is large and the ratio $d / \Lambda$ is very small, the chromatic dispersion curve is close to the material dispersion of silica. PCF with a large ratio $d / \Lambda$ and a small lattice constant $\Lambda$ has large normal dispersion in the $1.55 \mu \mathrm{m}$ wavelength range. The changing trend is well in agreement with that of the perfect hexagonal PCFs [24]. Also, we can see that the SC PCF with small lattice constant $\Lambda$ possesses two zero dispersion points within wavelengths ranging from 0.6 to $1.8 \mu \mathrm{m}$, and it is possible to tune the larger zero dispersion wavelength to visible to near-infrared regions by appropriately adjusting the structure parameters. However, it is difficult to control the nearly zero dispersion flattened in a wide wavelength range. Although the SC PCF with a large lattice constant $\Lambda$ and a very small $d / \Lambda$ can exhibit nearly zero dispersion-flattened behavior, the small ratio $d / \Lambda$ will lead to a large confinement loss.

Figure 8 shows the chromatic dispersion of the E-DC PCF as a function of wavelength for the incremental normalized parameter $a / \Lambda$ at $\Lambda=1.0$ and $1.6 \mu \mathrm{m}$, when the other parameters are set as $d / \Lambda=0.8$ and $\eta=3$. Compared with the chromatic dispersion of the SC PCF in Figure 7, the chromatic dispersion is apparently reduced by introducing the elliptical air hole in the core center, and the dispersion values in the whole wavelength window can be easily shifted to be negative by adjusting the size of the parameter $a / \Lambda$. When the lattice constant $\Lambda$ changes from 1.0 to $1.6 \mu \mathrm{m}$, the chromatic dispersion curve becomes more flattened in a wide wavelength range. Although the dispersion is slightly enlarged with increasing the lattice constant $\Lambda$, we can tune the normalized parameter $a / \Lambda$ to shift the curve up and down around zero. Therefore, the E-DC plays an important role in the flexible 


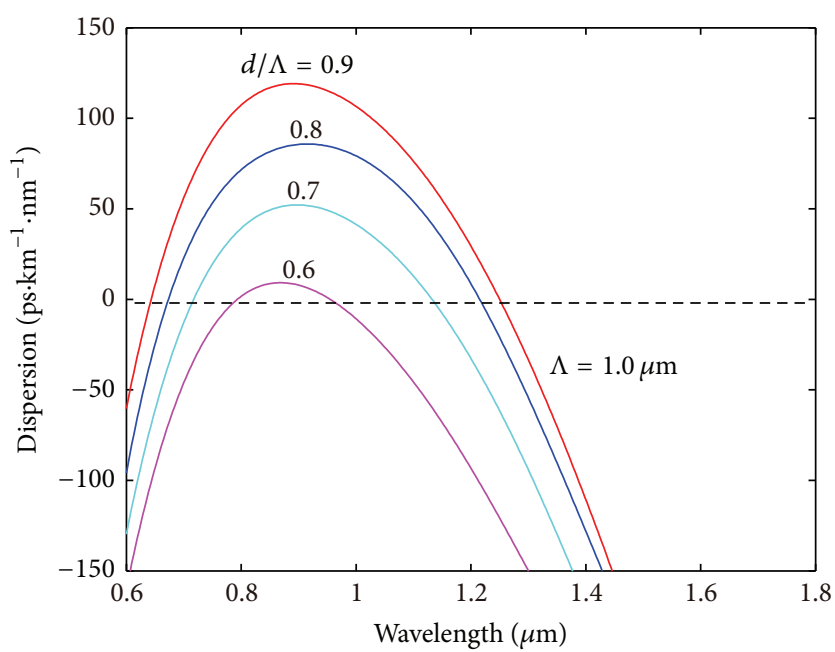

(a) $\Lambda=1.0 \mu \mathrm{m}$

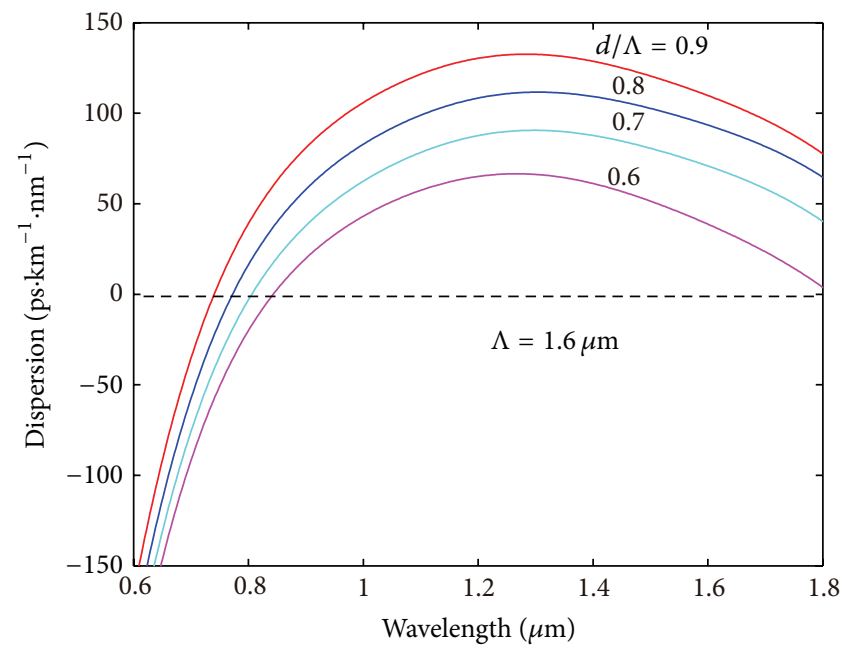

(b) $\Lambda=1.6 \mu \mathrm{m}$

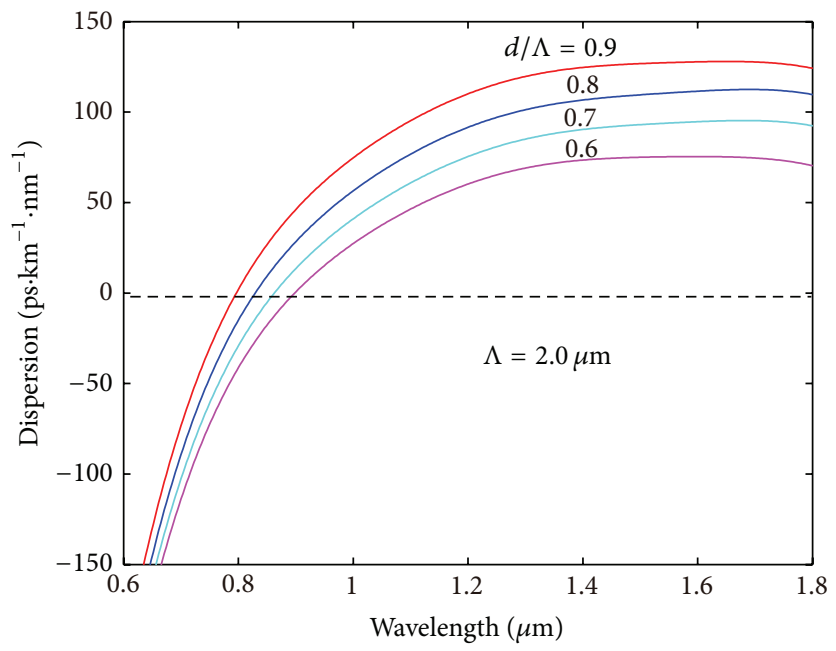

(c) $\Lambda=2.0 \mu \mathrm{m}$

FIGURE 7: Chromatic dispersion curves of the absolute SC PCF as a function of wavelength for incremental values of the normalized diameter $d / \Lambda$ ranging from 0.6 to 0.9 in steps of 0.1 at (a) $\Lambda=1.0 \mu \mathrm{m}$, (b) $\Lambda=1.6 \mu \mathrm{m}$, and (c) $\Lambda=2.0 \mu \mathrm{m}$.

adjustment of the dispersion and the dispersion slope to obtain a nearly zero dispersion-flattened PCF in a wide wavelength range. What is more, it should be noted that due to the large ratio $d / \Lambda$ of 0.8 the proposed E-DC PCF possesses the low confinement loss property. In a word, the proposed EDC PCF has the potential for obtaining negative and nearly zero flattened dispersion property, which makes it suitable as a chromatic dispersion controller, a dispersion compensator, or a candidate for nonlinear optical applications.

\section{Conclusion and Discussion}

To conclude our investigation, we have proposed a highly birefringent and nearly zero dispersion-flattened PCF based on the E-DC and pentagonal architecture. The simulated results indicate that the proposed PCF possesses an extraordinary electric field distribution where V-shaped notches appear in the core region due to the embedded elliptical air hole, and with the increase of wavelength the field power transfers from the surrounding silica into the central elliptical hole. From the refractive index distribution, the E-DC seems to be similar to the index-depressing doped core reported in $[25,26]$, which could be also used in the twin-core PCF for obtaining wavelength-independent coupling property. The mode field diameter is estimated to be about $2 \mu \mathrm{m}$, which implies the small effective mode area and highly nonlinear coefficient. Moreover, the modal birefringence is evaluated to be about $8 \times 10^{-3}$ at $\lambda=1.55 \mu \mathrm{m}$, which is larger than that of the equilateral pentagonal polarization maintaining PCF reported in [17-19]. In addition, in the proposed EDC PCF, we can tailor the parameters $a / \Lambda$ and $\Lambda$ to achieve the purpose of simultaneously tuning the dispersion and the dispersion slope. Compared with the SC PCF, the introduction of E-DC is helpful to control the chromatic dispersion to be negative or nearly zero flattened over a wide wavelength bandwidth. The high birefringence PCF may 


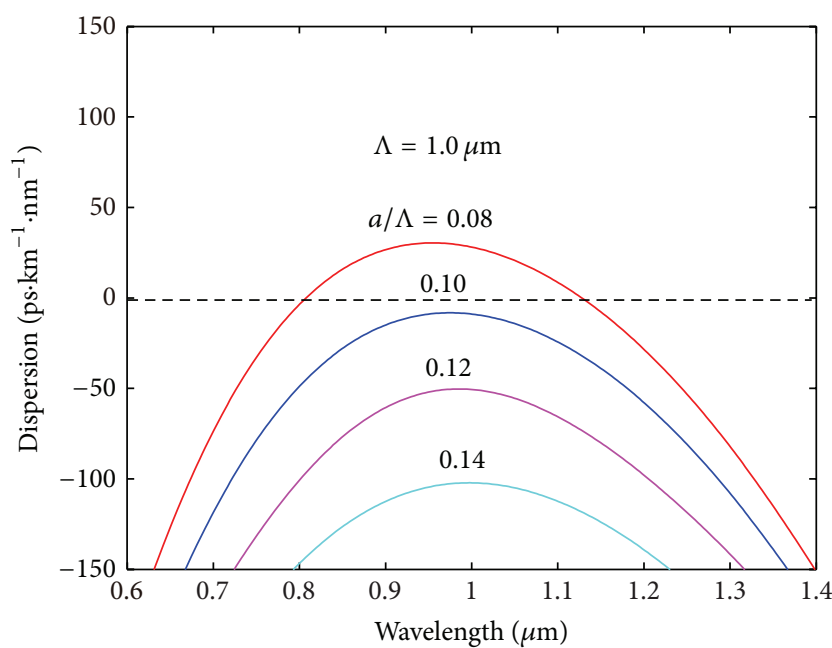

(a) $\Lambda=1.0 \mu \mathrm{m}$

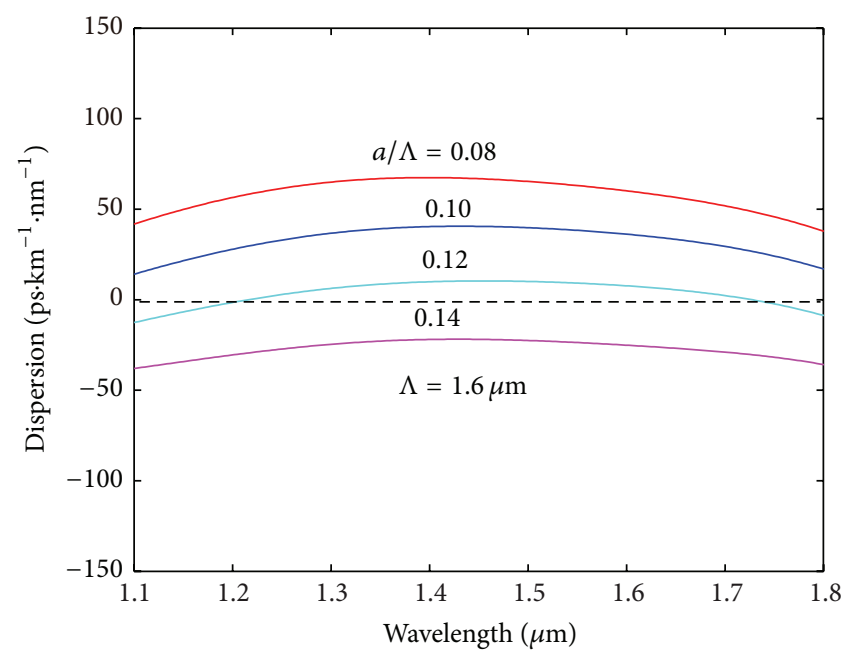

(b) $\Lambda=1.6 \mu \mathrm{m}$

FIGURE 8: Chromatic dispersion curves of the defected core PCF as a function of wavelength for incremental values of the parameter $a / \Lambda$ at (a) $\Lambda=1.0 \mu \mathrm{m}$ and (b) $\Lambda=1.6 \mu \mathrm{m}$. The other parameters are set as $d / \Lambda=0.8$ and $\eta=3$.

satisfy important applications in the field of optical fiber communications, fiber lasers, and fiber sensors, and the added negative or flattened dispersion feature makes it suitable as a chromatic dispersion controller, a dispersion compensator, or a candidate for nonlinear optical applications.

\section{Competing Interests}

The authors declare that there are no competing interests regarding the publication of this paper.

\section{Acknowledgments}

This work was supported by the Natural Science Foundation of China under Grant nos. 51379047 and 51379042.

\section{References}

[1] J. C. Knight, T. A. Birks, P. St. J. Russell, and D. M. Atkin, "Allsilica single-mode optical fiber with photonic crystal cladding," Optics Letters, vol. 21, no. 19, pp. 1547-1549, 1996.

[2] A. Ortigosa-Blanch, J. C. Knight, W. J. Wadsworth et al., "Highly birefringent photonic crystal fibers," Optics Letters, vol. 25, no. 18, pp. 1325-1327, 2000.

[3] W. Wang and B. Yang, "Characteristics analysis of photonic crystal fiber with rhombus air-core," Optik, vol. 123, no. 18, pp. 1669-1672, 2012.

[4] M. I. Hasan, M. Selim Habib, M. Samiul Habib, and S. M. Abdur Razzak, "Highly nonlinear and highly birefringent dispersion compensating photonic crystal fiber," Optical Fiber Technology, vol. 20, no. 1, pp. 32-38, 2014.

[5] F. Yu, Z. P. Wang, Y. G. Zhang, Y. K. Yu, and C. Y. Lv, "Analysis of a highly birefringent photonic crystal fiber with ellipserhombus air core," Optik, vol. 125, no. 20, pp. 6266-6269, 2014.

[6] T. P. Hansen, J. Broeng, S. E. B. Libori et al., "Highly birefringent index-guiding photonic crystal fibers," IEEE Photonics Technology Letters, vol. 13, no. 6, pp. 588-590, 2001.
[7] K. Saitoh and M. Koshiba, "Highly nonlinear dispersionflattened photonic crystal fibers for supercontinuum generation in a telecommunication window," Optics Express, vol. 12, no. 10, pp. 2027-2032, 2004.

[8] F. Poli, A. Cucinotta, S. Selleri, and A. H. Bouk, "Tailoring of flattened dispersion in highly nonlinear photonic crystal fibers," IEEE Photonics Technology Letters, vol. 16, no. 4, pp. 1065-1067, 2004.

[9] J. F. Liao, J. Q. Sun, M. D. Du, and Y. Qin, "Highly nonlinear dispersion-flattened slotted spiral photonic crystal fibers," IEEE Photonics Technology Letters, vol. 26, no. 4, pp. 380-383, 2014.

[10] M. Samiul Habib, M. Selim Habib, M. I. Hasan, S. M. A. Razzak, M. A. Hossain, and Y. Namihira, "Polarization maintaining large nonlinear coefficient photonic crystal fibers using rotational hybrid cladding," Optik, vol. 125, no. 3, pp. 1011-1015, 2014.

[11] T. Ritari, H. Ludvigsen, M. Wegmuller et al., "Experimental study of polarization properties of highly birefringent photonic crystal fibers," Optics Express, vol. 12, no. 24, pp. 5931-5939, 2004.

[12] K. Saitoh, M. Koshiba, T. Hasegawa, and E. Sasaoka, "Chromatic dispersion control in photonic crystal fibers: application to ultra-flattened dispersion," Optics Express, vol. 11, no. 8, pp. 843$852,2003$.

[13] D. J. Xu, H. S. Song, W. Wang, Y. Fan, and B. Yang, "Numerical analysis of a novel high birefringence photonic crystal fiber," Optik, vol. 124, no. 12, pp. 1290-1293, 2013.

[14] K. Saitoh, N. Florous, and M. Koshiba, "Ultra-flattened chromatic dispersion controllability using a defected-core photonic crystal fiber with low confinement losses," Optics Express, vol. 13, no. 21, pp. 8365-8371, 2005.

[15] A. Yin and L. Xiong, "Characteristics analysis of large solidcore square-lattice photonic crystal fibers with hybrid cladding," Optical Engineering, vol. 53, no. 1, Article ID 016112, 2014.

[16] M. Zhang, F. D. Zhang, Z. G. Zhang, and X. Chen, "Dispersionultra-flattened square-lattice photonic crystal fiber with small effective mode area and low confinement loss," Optik, vol. 125, no. 5, pp. 1610-1614, 2014. 
[17] X. Y. Li, H. R. Yang, Q. L. Zheng, J. H. Hao, and W. Hong, "Polarization and modal field properties of quinquangular-core photonic crystal fibers," Chinese Optics Letters, vol. 9, no. 9, Article ID 090602, 2011.

[18] X.-Y. Li, H.-R. Yang, and Y.-B. Gao, "High-birefringent photonic crystal fibers with small effective mode area," Journal of Chinese Inertial Technology, vol. 20, no. 6, pp. 715-719, 2012.

[19] H.-R. Yang, X.-Y. Li, W. Hong, and J.-H. Hao, "Equilateral pentagon polarization maintaining photonic crystal fibre with low nonlinearity," Chinese Physics B, vol. 21, no. 2, Article ID 024211, 2012.

[20] I. H. Malitson, "Interspecimen comparison of the refractive index of fused silica," Journal of the Optical Society of America, vol. 55, no. 10, pp. 1205-1209, 1965.

[21] K. Saitoh and M. Koshiba, "Full-vectorial imaginary-distance beam propagation method based on a finite element scheme: application to photonic crystal fibers," IEEE Journal of Quantum Electronics, vol. 38, no. 7, pp. 927-933, 2002.

[22] M. Koshiba and Y. Tsuji, "Curvilinear hybrid edge/nodal elements with triangular shape for guided-wave problems," Journal of Lightwave Technology, vol. 18, no. 5, pp. 737-743, 2000.

[23] F. Brechet, J. Marcou, D. Pagnoux, and P. Roy, "Complete analysis of the characteristics of propagation into photonic crystal fibers, by the finite element method," Optical Fiber Technology, vol. 6, no. 1, pp. 181-191, 2000.

[24] K. Saitoh and M. Koshiba, "Numerical modeling of photonic crystal fibers," Applied Optics, vol. 23, pp. 3580-3590, 2005.

[25] J. Leegsgaard, O. Bang, and A. Bjarklev, "Photonic crystal fiber design for broadband directional coupling," Optics Letters, vol. 29, no. 21, pp. 2473-2475, 2004.

[26] W. Lu, S. Lou, X. Wang, L. Wang, and R. Feng, "Ultrabroadband polarization splitter based on three-core photonic crystal fibers," Applied Optics, vol. 52, no. 3, pp. 449-455, 2013. 


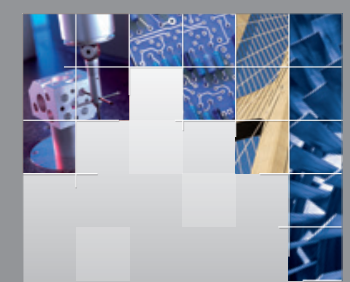

\section{Enfincering}
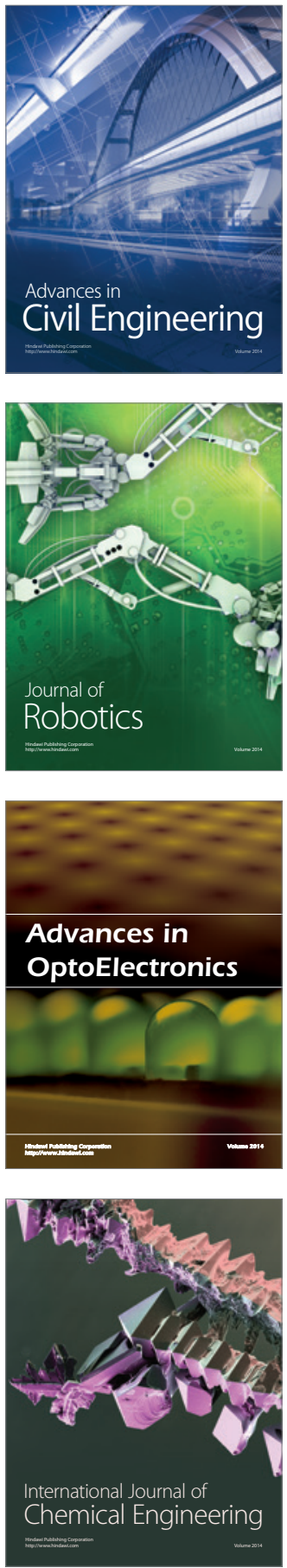

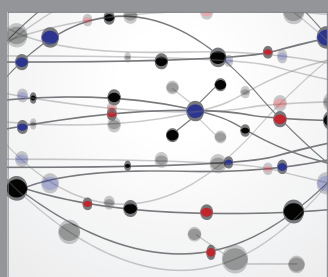

The Scientific World Journal

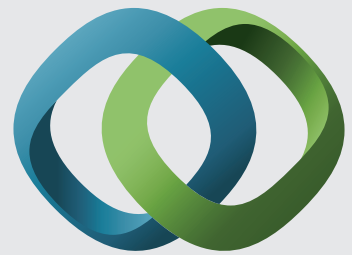

\section{Hindawi}

Submit your manuscripts at

http://www.hindawi.com
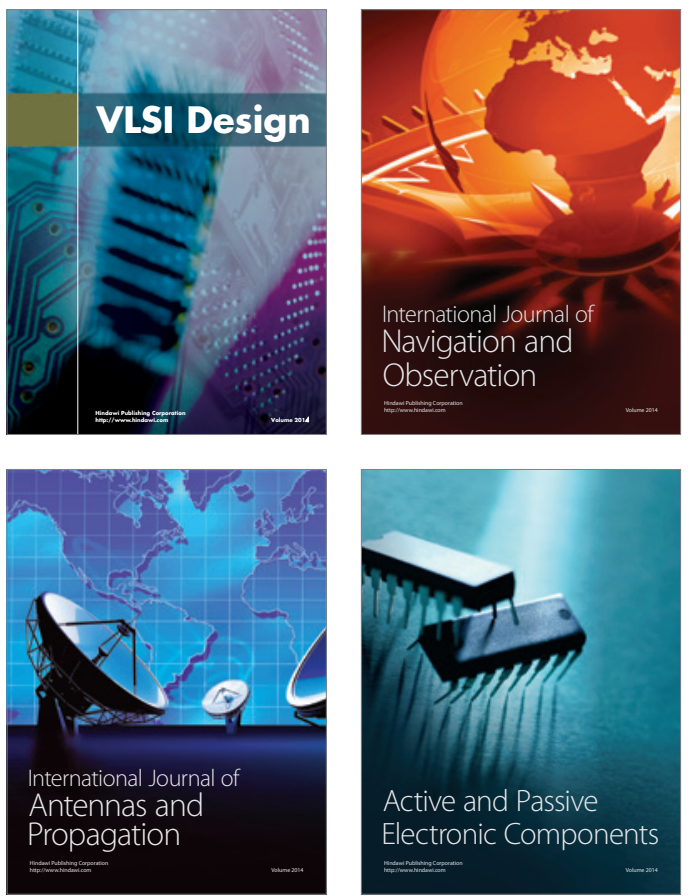
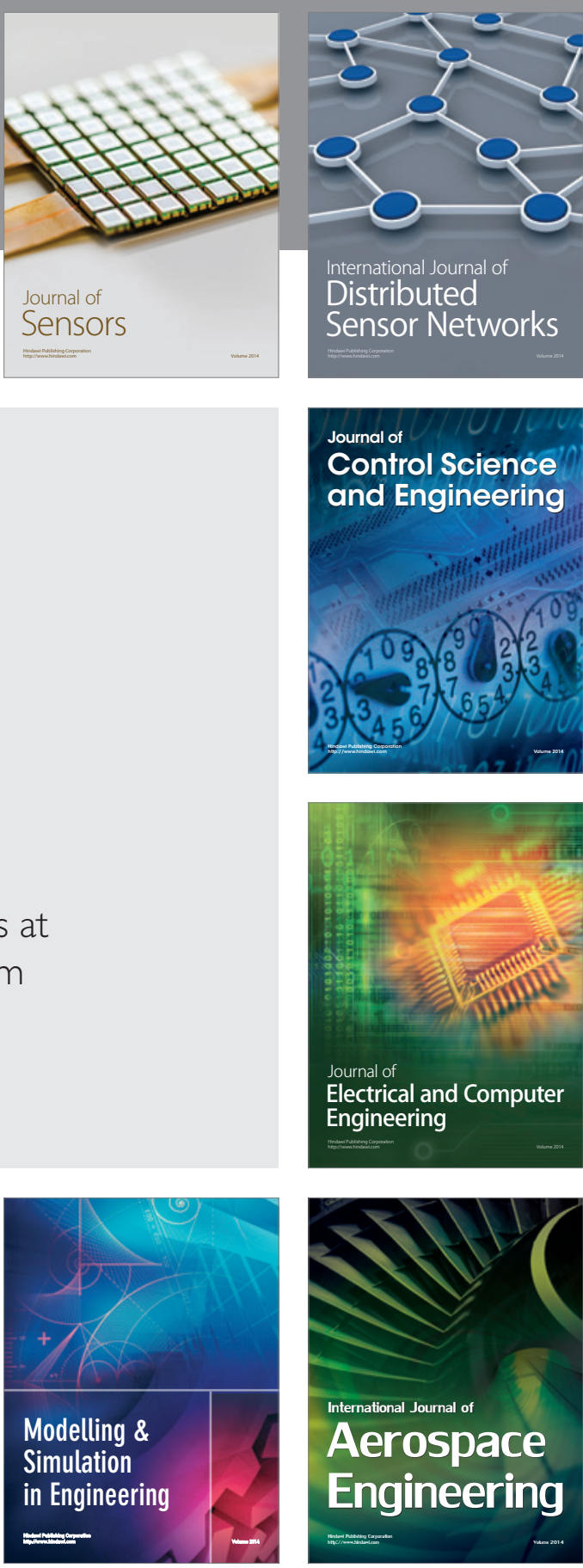

International Journal of

Distributed

Sensor Networks

Journal of

Control Science

and Engineering
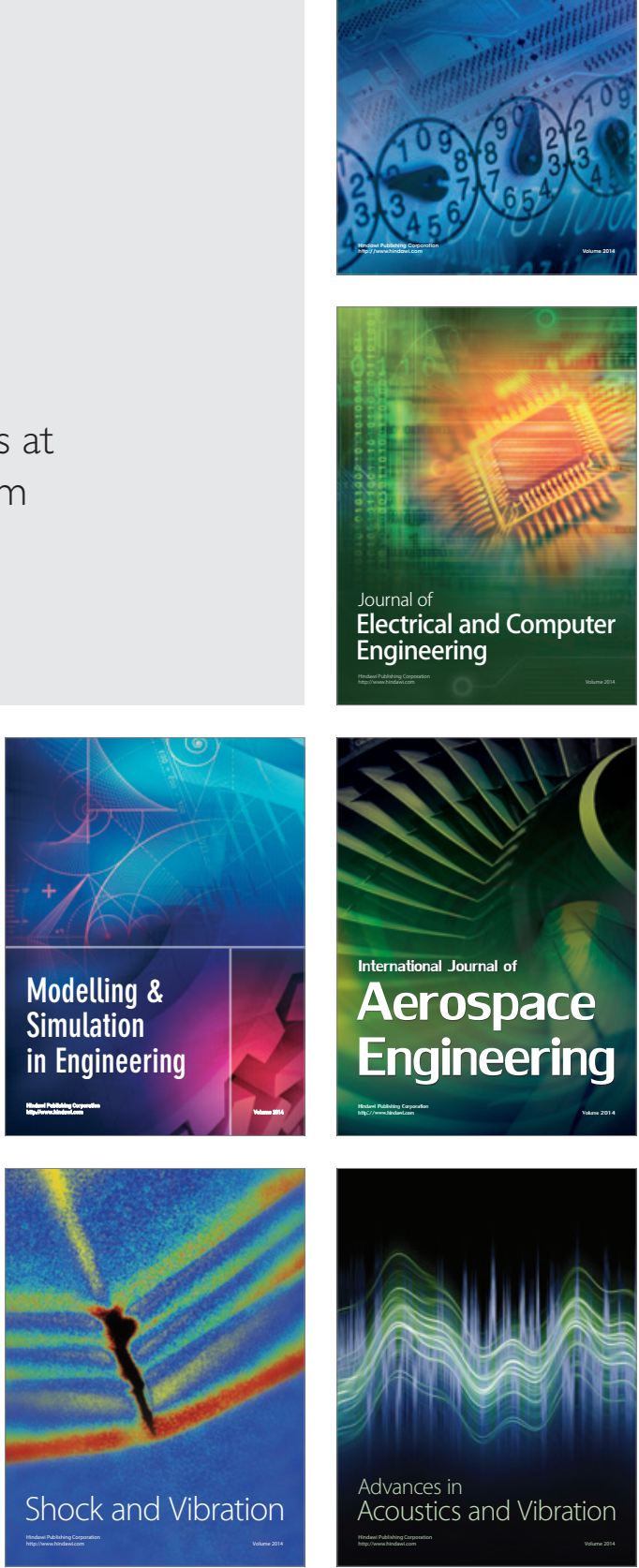